\title{
DERIVAÇÃO BILIODIGESTIVA NO TRATAMENTO DO TUMOR DE KLATSKIN
}

\author{
Intestinal biliary bypass in Klatskin's tumor treatment \\ Daniele Gehlen KLAUS, Diélly Cunha de CARVALHO, Marília Granzotto VOLPATO, \\ Alita Thizon MENEGALI, Jaime César Gelosa SOUZA, Orli FRANZON
}

ABCDDV/658

Klaus DG, Carvalho DC, Volpato MG, Menegali AT, Souza JCG, Franzon O. Derivação bileodigestiva no tratamento do tumor de klatskin. ABCD Arq Bras Cir Dig 2009;22(2):133-5

RESUMO - Introdução - Colangiocarcinomas são formas incomuns de neoplasia gastrointestinal, cuja incidência varia de $0,01 \%$ a $0,8 \%$. Os tumores de Klatskin são colangiocarcinomas hilares originados na bifurcação do ducto hepático principal. Atualmente a maioria dos tratamentos é paliativa. Objetivos - Relatar um caso de tumor de Klatskin submetido à drenagem cirúrgica da via biliar intra-hepática. Relato do caso - Homem de 68 anos, procurou assistência médica com quadro ictérico intenso e colangiorressonância magnética confirmou o diagnóstico de tumor de Klatskin. Levado à laparotomia exploradora verificou-se massa em hilo hepático com invasão portal, hepática e do complexo duodenopancreático. Optou-se por derivação biliodigestiva e hepatectomia parcial esquerda com anastomose hepaticojejunal. Houve boa evolução no pós-operatório, com diminuição da icterícia. Conclusão - A derivação biliodigestiva no tratamento do tumor de Klatskin é procedimento que alivia o quadro ictérico melhorando a qualidade de vida, e pode ser utilizado quando há irressecabilidade tumoral.

DESCRITORES - Tumor de Klatskin. Colangiocarcinoma hilar. Colangiojejunostomia. Drenagem.

\section{INTRODUÇÃO}

Colangiocarcinomas são formas incomuns de neoplasia gastrointestinal, cuja incidência varia de $0,01 \%$ a $0,8 \%{ }^{8}$. Os tumores de Klatskin (TK) são colangiocarcinomas hilares $(\mathrm{CCCH})^{16}$ originados na bifurcação do ducto hepático principal $^{9}$, cuja localização é extra-hepática. Correspondem a $25 \%$ de todos os colangiocarcinomas e são frequentemente adenocarcinomas ${ }^{5}$. Classificação de Longmire para as vias biliares extra-hepáticas, divide-os em tumores dos terços proximal, médio e distal ${ }^{8}$.

A clínica é principalmente icterícia obstrutiva ${ }^{6}$, além de dor abdominal em hipocôndrio direito e perda de peso ${ }^{7}$.

Os fatores de risco principais para o seu desenvolvimento são: cirrose, colangite esclerosante primária, coledocolitíase crônica, adenoma ductal, papilomatose biliar, doença de Caroli, cisto de colédoco e infestação parasitária biliar $^{12}$. Contudo, a maioria dos pacientes permanece com etiologia desconhecida ${ }^{12}$.

A letalidade é alta $^{13}$ e a ressecção cirúrgica é o tratamento de escolha; porém, a maioria está, no diagnóstico, em estágios avançados envolvendo estruturas adjacentes

Trabalho realizado na Universidade do Sul de Santa Catarina - UNISUL, Florianópolis, SC, Brasil

Endereço para correspondência: Daniele Gehlen Klaus, e-mail: daniklaus@terra.com.br e, por conseguinte, irressecáveis ${ }^{6,8,10,14}$. O prognóstico é pior nas lesões do terço superior, o que ocorre ente $40 \%$ e $60 \%$ dos $\operatorname{casos}^{6,15}$. Assim, a morbidade desse tumor atinge taxas de $30 \%$ a $50 \%$, enquanto que a mortalidade não ultrapassa $10 \%{ }^{15}$.

Derivação bilio-digestiva, após ressecção, é uma forma de reconstrução do trânsito, e a operação de Longmire é habitualmente utilizada. O procedimento de colangiojejunostomia intra-hepática ${ }^{9,16}$ usa o ducto hepático esquerdo distal para a anastomose bílio-digestiva.

\section{RELATO DO CASO}

Homem de 68 anos, natural e procedente de Itajaí, procurou assistência médica com quadro ictérico intenso e tomografia computadorizada demonstrando lesão na bifurcação dos ductos hepáticos com pequena dilatação das vias biliares e sem critérios de invasão adjacente ou metástases. Foi solicitada colangiorressonância magnética que confirmou os achados da tomografia.

Levado à laparotomia exploradora com incisão subcostal bilateral, verificou-se massa em hilo hepático com invasão portal, hepática e do complexo duodenopancreático. Sendo contraindicada a ressecção, optou-se por derivação biliodigestiva e hepatectomia parcial esquerda com anastomose hepaticojejunal (Figura 1). Houve boa evolução no pós-operatório, com diminuição da icterícia. 


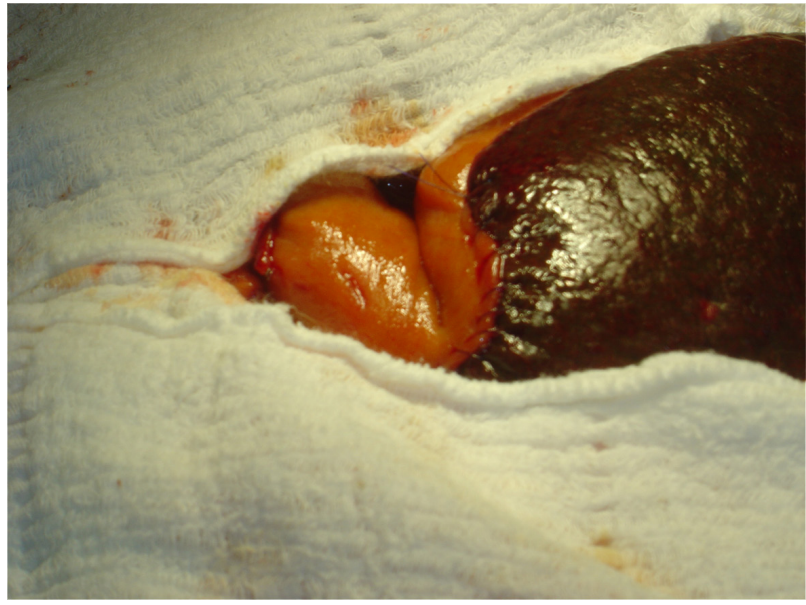

FIGURA 1 - Trans-operatório da derivação biliodigestiva com visualização final da anastomose

\section{DISCUSSÃO}

Os critérios para ressecabilidade ${ }^{2}$ do TK são: 1) infiltração da bifurcação dos ductos hepáticos; 2) envolvimento da artéria hepática comum juntamente com artéria hepática direita ou esquerda; e 3) envolvimento da veia porta simultaneamente. $\mathrm{O}$ exame ecográfico com doppler no pré-operatório tem acurácia diagnóstica do envolvimento da veia porta de $81,82 \%{ }^{2}$ e também é alta a da colangioressonância e angiografia-MDCT. São eles os métodos de escolha para o pré-operatório desse tumor ${ }^{2}$.

Grande preocupação quanto à ressecção hepática nos colangiocarcinomas hilares reside na quantidade de tecido hepático saudável que permanece depois da remoção de porções extensas de fígado acometido pela doença. Nesse sentido Vogl et al. ${ }^{11}$ descrevem que ressecções de mais de $65 \%$ do fígado, aumentam muito o risco de falha pós- operatória por insuficiência hepática, o que pode ser evitado pela embolização arterial realizada no pré-operatório ${ }^{11}$. O seguimento no pós-operatório com imagens de tomografia computadorizada comprovaram aumento do volume do parênquima saudável do lobo esquerdo, ou seja, ocorre regeneração com hipertrofia do parênquima hepático ${ }^{11}$.

Autores 15 defendem o transplante hepático como boa opção para os colangiocarcinomas hilares irressecávis ${ }^{8,16}$. Outros o contraindicam, por considerarem os índices de recidiva do tumor muito altos 1.

Liu et al. $^{4}$ analisam o uso de colangiopancreatografia endoscópica retrógrada (CPER) e da inserção de próteses endoscópicas como maneira de drenar a via biliar no préoperatório e aliviar os sintomas, ou mesmo como tratamento paliativo nos casos de irressecabilidade do tumor. Obtiveram sucesso na drenagem em $41 \%$ dos pacientes, com morbidade e mortalidade relacionadas ao procedimento de $25 \%$ e $5 \%$, respectivamente ${ }^{4}$. Weber et al. ${ }^{12}$ indica a colangiografia endoscópica retrógrada e drenagem percutânea trans-hepática como sendo medidas seguras e efetivas, com poucas complicações para a drenagem da via biliar em condições de irressecabilidade. Mansfield et al. ${ }^{6}$ mostram o uso de endopróteses biliares como forma de aliviar os sintomas, configurando tratamento paliativo ou adjuvante à cirurgia com mortalidade no hospitalar de até $17 \%$ e morbidade de até $50 \%{ }^{6}$. A drenagem biliar endoscópica apresentou altos índices de falha, necessitando subsequente drenagem percutânea ${ }^{6}$.

\section{CONCLUSÕES}

A derivação biliodigestiva no tratamento do tumor de Klatskin é procedimento que alivia o quadro ictérico do paciente melhorando sua qualidade de vida, e pode ser utilizado quando há irressecabilidade tumoral.

Klaus DG, Carvalho DC, Volpato MG, Menegali AT, Souza JCG, Franzon O. Intestinal biliary bypass in Klatskin's tumor treatment. ABCD Arq Bras Cir Dig 2009;22(2):133-5

ABSTRACT - Introduction - Cholangiocarcinomas are uncommon (0,01 to 0,8\%) forms of gastrointestinal neoplasms. The Klatskin tumour is ductal cholangiocarcinoma originated on the bifurcation of main hepatic duct. Currently, the majority of cases are treated in palliative way. Case reportMan with 68 years old seek medical assistance with intense jaundice and MRI gave the final diagnosis of Klatskin tumour. Exploratory laparotomy showed big hepatic mass involving portal vein and neighboring tissues. He was submitted to palliative procedure with hepatojejunal anastomosis and partial hepatectomy. He was discharged from the hospital with better clinical conditions and alleviated from his jaundice. Conclusion - Intestinal biliary bypass in Klatskin is an indicated procedure to alleviate the jaundice in the terminal phase of this tumour, improving the quality of live.

HEADINGS - Klatskin's tumor. Ductal cholangiocarcinoma. Cholangiojejunostomy. Drainage.

\section{REFERÊNCIAS}

1. Becker G, Momm F, Schwacha H, Hodapp N, Usadel H, Gleiber M, Barke A, Schmitt-Graff A, Henne K, Blum HE. Klatskin tumor treated by interdisciplinary therapies including stereotactic radiotherapy: a case report. World Journal of Gastroenterology 2005; 11(31):4923-4926.

2. Chen HW, Pan AZ, Zhen ZJ, Su SY, Wang JH, Yu SCH, Lau WI. Preoperative evaluation of resectability of Klatskin tumor with 16-MDCT angiography and cholangiography. AJR 2006; 186:1580-1586.
3. Hann LE, Greatex KV, Bach AM, Fong Y, Blumgart LH. Cholangiocarcinoma at the hepatic hilus: sonographic findings. AJR 1997; 168:985-989.

4. Liu C, Lo C, Lai ECS, Fan S. Endoscopic retrograde cholangiopancreatography and endoscopic endoprosthesis insertion in patients with Klatskin tumors. Arch Surg 1998; 133:293-296.

5. Long-Lin Y, Bin S, Juan X, Ying-Chun L. Hilar cholangiocarcinoma: preoperative evaluation with a three dimensional volumetric interpolated breath-hold examination magnetic resonance imaging sequence. Chinese Medical Journal 2007; 120(8):636-642. 
6. Mansfield SD, Barakat O, Charnley RM, Jaques BC, O'Suilleabhain CB, Atherton PJ, Manas D. Management of hilar cholangiocarcinoma in the North of England: pathology, treatment, and outcome. World Journal of Gastroenterology $2005 ; 11(48): 7625-7630$.

7. Schmeding M, Neumann U, Neuhaus P. Colonic metastasis of Klatskin tumor: case report and discussion of the current literature. World Journal of Gastroenterology 2006; 12(33):5393-5395.

8. Shimoda M, Farmer DG, Colquhoun SD, Rosove M, Ghobrial RM, Yersiz H, Chen P, Busuttil RW. Liver transplantation for cholangiocellular carcinoma: analysis of a single-center experience and review of the literature. Liver transplantation 2001; 7(12):1023-1033.

9. Torres OJM, Barros CA, Barros NDC, Melo LAL, Ferry JM, Ribas Filho JM, Czeczko NG, Matias JEF. Tratamento cirúrgico do tumor de Klatskin. ABCD Arq Bras Cir Dig 2003; 16(2):

10. Utrillas AC, Llado L, Alba E, Valls C, Cruz M, Figueras J, Serrano T, Ramos E, Torras J, Rafecas A. Ictericia obstructive secundaria a colangitis linfoplasmocitaria. Diagnóstico diferencial con el tumor de Klatskin. Rev Esp Enferm Dig 2005; 97(12):917-926.
11. Vogl TJ, Balzer JO, Dette K, Hintze R, Pegios W, Maurer J, Keck H, Neuhaus P, Felix R. Inicially unresectable hilar cholangiocarcinoma: hepatic regeneration after transarterial embolization. Radiology 1998; 208(1):217-222.

12. Weber A, Landrock S, Schneider J, Stangl M, Neu B, Born P, Classen M, Rosch T, Schmid RM, Prinz C. Long-term outcome and prognostic factors of patients with hilar cholangiocarcinoma. World Journal of Gastroenterology 2007; 13(9):1422-1426.

13. Welzel T, Mcglynn KA, Hsing AW, O'brien TR, Pfeiffer RM. Impact of classification of hilar cholangiocarcinomas (Klatskin tumors) on the incidence of intra- and extrahepatic cholangiocarcinoma in the United States. Journal of the Nacional Cancer Institute 2006; 98(12):873-875.

14. Yi B, Zhang BH, Zhang YJ, Jiang XQ, Zhang BH, Yu WL, Chen QB, Wu MC. Surgical procedure and prognosis of hilar cholangiocarcinoma. Hepatobiliary and Pancreatic Diseases International 2004; 3(3):453-457.

15. Zhang BH, Cheng QB, Luo XJ, Zhang YJ, Jiang XQ, Zhang BH, Yi B, Yu WL, Wu MC. Surgical therapy for hiliar cholangiocarcinoma: analysis of 198 cases. Hepatobiliary and Pancreatic Diseases International 2006; 5(2):278-282.

16. Zheng SS, Shi QF, Liang TB, Wu J, Wang WL, Shen Y, Zhang M. Orthotopic liver transplantation for patients with Klatskin tumor. Hepatobiliary and Pancreatic Diseases International 2005; 4(1):28-31.

Fonte de financiamento: não há Conflito de interesse: não há Recebido para publicação: 21/01/2009 Aceito para publicação: 23/03/2009 